\title{
Parametric electron pumping through a quantum dot in the Kondo regime
}

\author{
Baigeng Wang and Jian Wang \\ Department of Physics, The University of Hong Kong, Pokfulam Road, Hong Kong, China \\ (Received 30 October 2001; revised manuscript received 22 January 2002; published 10 June 2002)
}

\begin{abstract}
We report a theoretical analysis of parametric electron pump through a quantum dot in the Kondo regime. In the adiabatic regime, we have derived the expression for pumped current in the Kondo regime using nonequilibrium Green's function. The pumped current versus different system parameters such as gate voltage, pumping amplitude, as well as the phase difference between two pumping forces are calculated and interesting physics are revealed.
\end{abstract}

DOI: 10.1103/PhysRevB.65.233315

PACS number(s): 73.23.Ad, 73.40.Gk

The general physics of parametric electron pump has been the subject of recent studies. ${ }^{1-15}$ It is in particular inspired by the recent experiment of Switkes et al. In this experiment, ${ }^{3}$ the pumped current through an open quantum dot is driven by two gates with oscillating voltages controlling the deformation of the shape of the dot. The pumped dc voltage $V_{d o t}$ is measured to vary with the phase difference $\phi$ between the two gate voltages, and is antisymmetric about $\phi=\pi$. At low pumping amplitude the experimental data gave $V_{d o t} \sim \sin \phi$. In the strong pumping regime, the dependence of $V_{d o t}$ on $\phi$ becomes nonsinusoidal showing $V_{d o t}(0) \neq 0$, whereas keeping $V_{d o t}(\pi) \approx 0$ for all pumping strength. Many of these experimental findings have been explained theoretically. However, apart from Refs. 9 and 10, to date most of the theoretical investigations of parametric pumping have assumed single electron approximation. It would be interesting to see how the strong electron-electron interaction modifies the pumped current. For this purpose, we report in this paper a theoretical analysis of the parametric electron pump through the quantum dot in the Kondo regime using adiabatic theory. Our results indicate, in the Kondo regime, that the general behavior of the pumped current is similar to that of the conductance. Above the Kondo temperature, as one scans the gate voltage $v_{g}$ we found two peaks in the pumped current corresponding to the resonant tunneling peak and Coulomb charging peak. When the temperature is below the Kondo temperature, a new peak in the pumped current starts to emerge at $v_{g}=-U / 2$ in the middle of the resonant peak and Coulomb charging peak. At zero temperature, the pumped current has a broad peak at $v_{g}=-U / 2$ which is the superposition of these three peaks. This is very different from the noninteracting case where there is only one peak in the pumped current. In the Kondo regime, we found that as one varies the pumping amplitude, the pumped current increases quadratically for small amplitude and then scales linearly with the pumping amplitude. Our result also shows that the pumped current is antisymmetric about $\phi=\pi$ and is a nonsinusoidal function of $\phi$ for large pumping amplitude. Our result suggests that the Kondo signature can also be found in the pumped current which can be checked experimentally.

We consider a two-dimensional (2D) quantum dot with leads connected to the dot through narrow constrictions controlled by gate voltages. Since the threshold of electron propagation in the constriction may be lower than that in the lead, the constrictions act like a double barrier whose height can be tuned by two gate voltages. The cyclic variation of these two pumping gate voltages allow the parametric electron pumping through the quantum dot. To simplify the calculation, we use the one-dimensional double barrier potential to model the quantum dot.

To analyze parametric quantum pumping, we make use of the nonequilibrium Green's-function method. Using the distribution function, the total charge in the system during the pumping is given by $Q(x, t)=-i e \int(d E / \pi)$ $\times\left[G^{<}(E,\{X(t)\})\right]_{x x}$ where $G^{<}$is the lesser Green's function in real space, $x$ labels the position, and $\{X(t)\}$ describes a set of external parameters which facilitates the pumping process. $G^{<}$is related to the retarded and advanced Green's functions $G^{r}$ and $G^{a},{ }^{16} G^{<}(E,\{X\})=-f(E)\left[G^{r}(E,\{X\})\right.$ $\left.-G^{a}(E,\{X\})\right]$ where the retarded Green's function in real space is given by

$$
G^{r}(E,\{X\})=\frac{1}{E-H-V_{p}-\Sigma^{r}} .
$$

In Eq. (1), $\Sigma^{r}$ is the self-energy and $V_{p}$ is a diagonal matrix describing the variation of the potential landscape due to the external pumping parameter $X$. In order for a parametric electron pump to function, we need simultaneous variation of two system parameters $X_{1}(t)=X_{10}+X_{1 p} \sin (\omega t)$ and $X_{2}(t)$ $=X_{20}+X_{2 p} \sin (\omega t+\phi)$. Hence, in our case, the potential due to the gates can be written as $V_{p}=X_{1} \Delta_{1}+X_{2} \Delta_{2}$, where $\boldsymbol{\Delta}_{i}$ is potential profile for each gate. If the time variation of these parameters are slow, i.e., for $X(t)=X_{0}+\delta X \sin (\omega t)$, then the charge of the system coming from all contacts due to the infinitesimal change of the system parameter $(\delta X \rightarrow 0)$ is

$$
d Q(t)=\sum_{i} \partial_{X_{i}} \operatorname{Tr}[Q(x, t)] \delta X_{i}(t)
$$

where $\operatorname{Tr}[\cdots]$ is over the positions. It is easily seen that the total charge in the system in a period is zero which is required for the charge conservation. To calculate the pumped current, we have to find the charge $d Q_{\alpha}$ passing through contact $\alpha$ due to the change of the system parameters. Using the Dyson equation $\partial_{X_{i}} G^{r}=G^{r} \boldsymbol{\Delta}_{i} G^{r}$, Eq. (2) becomes 


$$
\begin{aligned}
d Q(t) & =\frac{i e}{\pi} \sum_{j} \int d E \operatorname{Tr}\left[G^{r} \boldsymbol{\Delta}_{j} G^{r}-\text { c.c. }\right] f(E) \delta X_{j}(t) \\
& =\frac{e}{\pi} \int d E\left(\partial_{E} f\right) \sum_{j} \operatorname{Tr}\left[G^{r} \Gamma G^{a} \boldsymbol{\Delta}_{j}\right] \delta X_{j}(t),
\end{aligned}
$$

where we have used the fact that $G^{r} \Gamma G^{a}=i\left(G^{r}-G^{a}\right)$ and $\Gamma=\Sigma_{\alpha} \Gamma_{\alpha}$ is the linewidth function. So we obtain

$$
d Q_{\alpha}(t)=\frac{e}{\pi} \int d E\left(\partial_{E} f\right) \sum_{j} \operatorname{Tr}\left[G^{r} \Gamma_{\alpha} G^{a} \boldsymbol{\Delta}_{j}\right] \delta X_{j}(t) .
$$

Furthermore, the current flowing through contact $\alpha$ due to the variation of parameters $X_{1}$ and $X_{2}$, in one period of time, is given by

$$
I_{\alpha}=\frac{1}{\tau} \int_{0}^{\tau} d t d Q_{\alpha} / d t,
$$

where $\tau=2 \pi / \omega$ is the period of cyclic variation. In terms of injectivity ${ }^{17}$ given by ${ }^{18}$

$$
\frac{d N_{\alpha}}{d X_{j}}=\int \frac{d E}{2 \pi}\left(\partial_{E} f\right) \operatorname{Tr}\left[G^{r} \Gamma_{\alpha} G^{a} \boldsymbol{\Delta}_{j}\right]
$$

Eq. (5) reduces to the familiar formula ${ }^{2}$

$$
I_{\alpha}=\frac{e \omega}{\pi} \int_{0}^{\tau} d t\left[\frac{d N_{\alpha}}{d X_{1}} \frac{d X_{1}}{d t}+\frac{d N_{\alpha}}{d X_{2}} \frac{d X_{2}}{d t}\right] .
$$

Note that Eq. (7) is a general expression applicable to the case of interacting and noninteracting systems as long as the retarded Green's function is known.

For the transport in the Kondo regime, we consider the following Hamiltonian $H=H_{0}+H_{I}+H_{T}$ with

$$
\begin{gathered}
H_{0}=\sum_{k \alpha \sigma} \epsilon_{k \alpha} C_{k \alpha \sigma}^{+} C_{k \alpha \sigma}+\sum_{\sigma m}\left[E_{m}+v_{g}\right] d_{\sigma m}^{\dagger} d_{\sigma m}, \\
H_{I}=U \sum_{m} n_{m \uparrow} n_{m \downarrow},
\end{gathered}
$$

and

$$
H_{T}=\sum_{k \sigma m \alpha} T_{k \alpha m} C_{k \alpha \sigma}^{\dagger} d_{\sigma m}+\text { c.c., }
$$

where $C_{k \alpha \sigma}^{\dagger}$ is the creation operator of lead $\alpha$ and $d_{\sigma m}^{\dagger}$ is the creation operator of the scattering regime at energy level $\mathrm{m}$. We have applied the gate voltage $v_{g}$ to control the energy level in the scattering region. For this Hamiltonian $\Gamma_{\alpha}$ defined in Eq. (4) is given by $\left(\Gamma_{\alpha}\right)_{m n}=2 \pi \Sigma_{k} T_{k \alpha m}^{*} T_{k \alpha n} \delta(E$ $\left.-\epsilon_{k \alpha}\right)$. There are many approaches to treat the scattering problem in the Kondo regime. ${ }^{19-23}$ We find it is convenient to use the perturbation scheme proposed by Levy Yeyati et $a l^{19}$ and Kajueter and Kotliar. ${ }^{20}$ In this approach, the retarded Green's function is given by

$$
G^{r}=\frac{1}{E-H-\Sigma_{l e a d}^{r}-\Sigma_{s}^{r}},
$$

where $\Sigma_{\text {lead }}^{r}$ is the self-energy due to the coupling between the scattering region and leads. The effect of strongly electron-electron interaction is included in the self-energy $\sum_{s}^{r}, 19,20,24$

$$
\Sigma_{s}^{r}(E)=U n+\frac{A \Sigma_{0}^{r}(E)}{1-B \Sigma_{0}^{r}(E)},
$$

where $\Sigma_{0}^{r}$ is the self-energy due to the second order contribution in $U$,

$$
\begin{aligned}
\Sigma_{0}^{r}(E)= & \frac{i U^{2}}{8 \pi^{3}} \int \frac{d E_{1} d E_{2} d E_{3}}{E+E_{3}-E_{1}-E_{2}+i \delta} \\
& \times\left[G_{0}^{>}\left(E_{1}\right) G_{0}^{>}\left(E_{2}\right) G_{0}^{<}\left(E_{3}\right)\right. \\
& \left.-G_{0}^{<}\left(E_{1}\right) G_{0}^{<}\left(E_{2}\right) G_{0}^{>}\left(E_{3}\right)\right],
\end{aligned}
$$

where $G_{0}^{r}=1 /\left(E-H_{0}-\Sigma_{\text {lead }}^{r}\right)$ and $G_{0}^{<}=-f\left(G_{0}^{r}-G_{0}^{a}\right)$. Here for simplicity, we have only considered a particular energy level $E_{0}$ and used the wideband limit. ${ }^{16}$ The coefficients $A$ and $B$ in Eq. (12) are determined by the solutions in two limiting cases: large energy limit and atomic limit, ${ }^{20}$ from which we have $A=[n(1-n)] /\left[n_{0}\left(1-n_{0}\right)\right]$ and $B$ $=[(1-2 n)] /\left[n_{0}\left(1-n_{0}\right) U\right]$ with $n=-\int d E f(E) \operatorname{Im} G^{r} / \pi$ is the physical particle number and $n_{0}=-\int d E f(E) \operatorname{Im} G_{0}^{r} / \pi$ is the fictitious particle number. This scheme gives a good description for the case of half filling. Away from that, one must replace $H_{0}$ in $G_{0}^{r}$ and $G^{r}$ by a self-consistent Hamiltonian $H_{\text {eff }}$ and use the Friedel sum rule ${ }^{19,20}$

$$
n=\frac{1}{2}-\frac{1}{\pi} \arctan \left(\frac{E+\Sigma_{s}^{r}+\operatorname{Re} \Sigma_{\text {lead }}^{r}}{\operatorname{Im} \Sigma_{\text {lead }}^{r}}\right) .
$$

The self-consistent solution of Eqs. (11), (12), and (14) determines the self-energy $\Sigma_{s}^{r}$ which will be used in the calculation of pumped current. We now apply Eq. (5) to calculate the pumped current in the Kondo regime. The double barrier structure is modeled by potential $U(x)=X_{1} \delta(x+a / 2)$ $+X_{2} \delta(x-a / 2)$ where $X_{1}$ and $X_{2}$ are barrier heights which vary in a cyclic fashion to allow the charge pumping. In particular, we set $X_{i}=v_{0}+v_{p} \sin \left(\omega t+\phi_{i}\right)$ with $\phi_{1}=0$ and $\phi_{2}=\phi$. We will fix the units by setting $\hbar=2 m=1$ in the following analysis. For the GaAs system with $a=1000 \mathrm{~A}$, the energy uint is $E=56 \mu \mathrm{eV}$. We will also fix the on site potential $U=5$ which is much smaller than the level spacing in the quantum dot, frequency $\omega=1$, the barrier height $v_{0}$ $=79.2$, and phase difference $\phi=\pi / 2$ (unless specified otherwise). Finally, the energy of incoming electron is chosen to be in line with a resonant level $E_{0}$ when $v_{g}=0$. In Fig. 1 we present the transmission coefficient versus gate voltage (which controls the levels in the quantum dot) at different temperatures $T$. When the temperature is higher than the Kondo temperature $T_{k}=0.02$ (dashed line in Fig. 1), we see two peaks: resonant tunneling peak at $v_{g}=0$ (for $E=E_{0}$ ) and the Coulomb charging peak at $v_{g}=-U\left(E=E_{0}+U\right)$. At low temperatures below $T_{k}$, the co-tunneling process leads to a new peak, the Kondo peak, at the Fermi level. As the temperature is lowered, the peak height of Kondo peak in- 


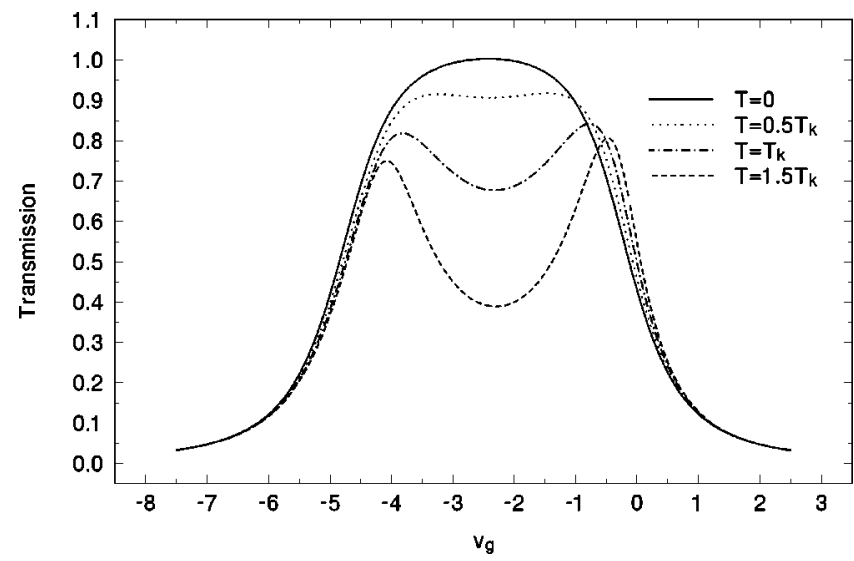

FIG. 1. The transmission coefficient versus gate voltage at different temperatures.

creases and the dip between resonant peak and charging peak diminishes. At zero temperature, the broad peak at $v_{g}$ $=-U / 2$ in Fig. 1 is the superposition of these three peaks. For $v_{g}>0$ or $v_{g}<-U$, the transmission coefficient is almost temperature independent. Note that the peak heights (near $v_{g}=0$ and $v_{g}=-U$ ) are asymmetric about the $v_{g}=-U / 2$. This is because the linewidth function $\Gamma$ depends on energy or in our case depends on the gate voltage. Figure 2 depicts the pumped current as a function of gate voltage at different temperatures and for different pumping amplitudes. Generally speaking, the pumped current follows similar pattern of the transmission coefficient at different temperatures due to the fact that the pumped current is proportional to the density of states of the system which also manifests in the transmission coefficient. We see that as the pumping amplitude becomes larger, the ratio $I_{p}\left(T=0, v_{g}=-U / 2\right) / I_{p}(T$ $=1.5 T_{k}, v_{g}=-U / 2$ ) becomes smaller (see Fig. 3 for further discussion); at $T \neq 0$ two resonant peaks become broader and move away from each other; the Kondo peak at $T=0$ becomes broader and flattened. We also notice that the pumped current increases as the pumping amplitude increases. In particular, as the pumping amplitude increases, the peak height of resonant states at $E_{0}$ and $E_{0}+U$ increases much faster

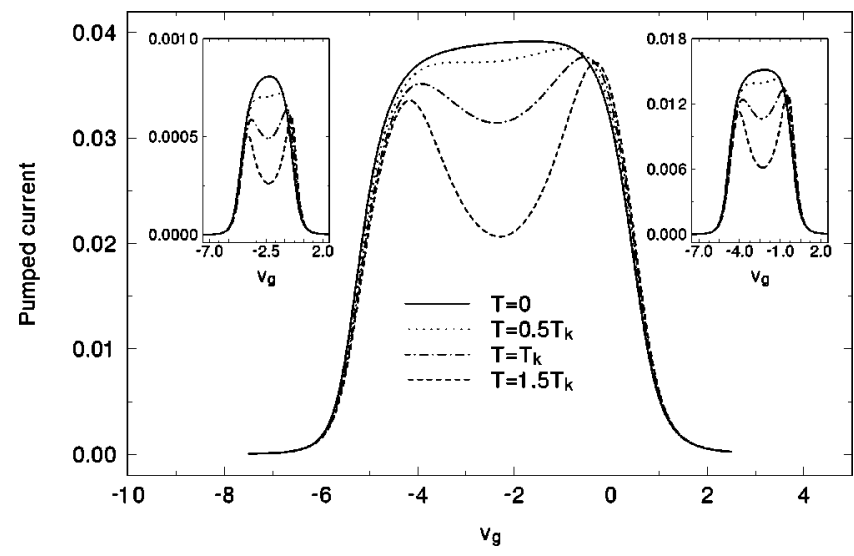

FIG. 2. The pumped current versus gate voltage for different pumping amplitudes $v_{p}$. Main figure: $v_{p}=0.1 v_{0}$; left inset: $v_{p}$ $=0.01 v_{0}$; right inset: $v_{p}=0.05 v_{0}$.

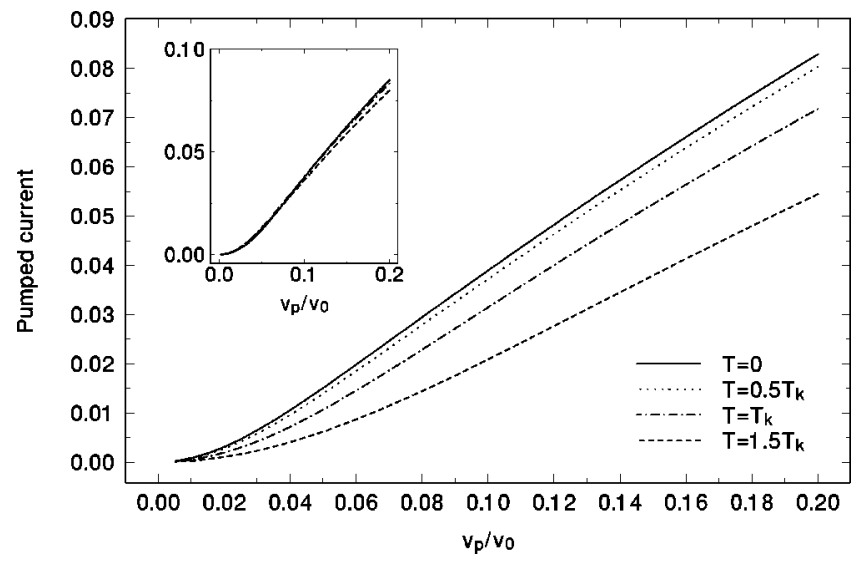

FIG. 3. The pumped current versus relative pumping amplitude at different gate voltages. Main figure: $v_{g}=-2.5$; inset: $v_{g}=-0.48$.

than that of the Kondo peak and when $v_{p}=0.1 v_{0}$ they have almost the same height. In Fig. 3 we plot the pumped current versus relative pumping amplitude $v_{p} / v_{0}$ at two different gate voltages: one at $v_{g}=-U / 2$ and the other near the resonant level when $v_{g}=-0.5$. At the $v_{g}=-U / 2$, the dependence on the relative pumping amplitude shows the expected quadratic behavior for small amplitude since the pumped
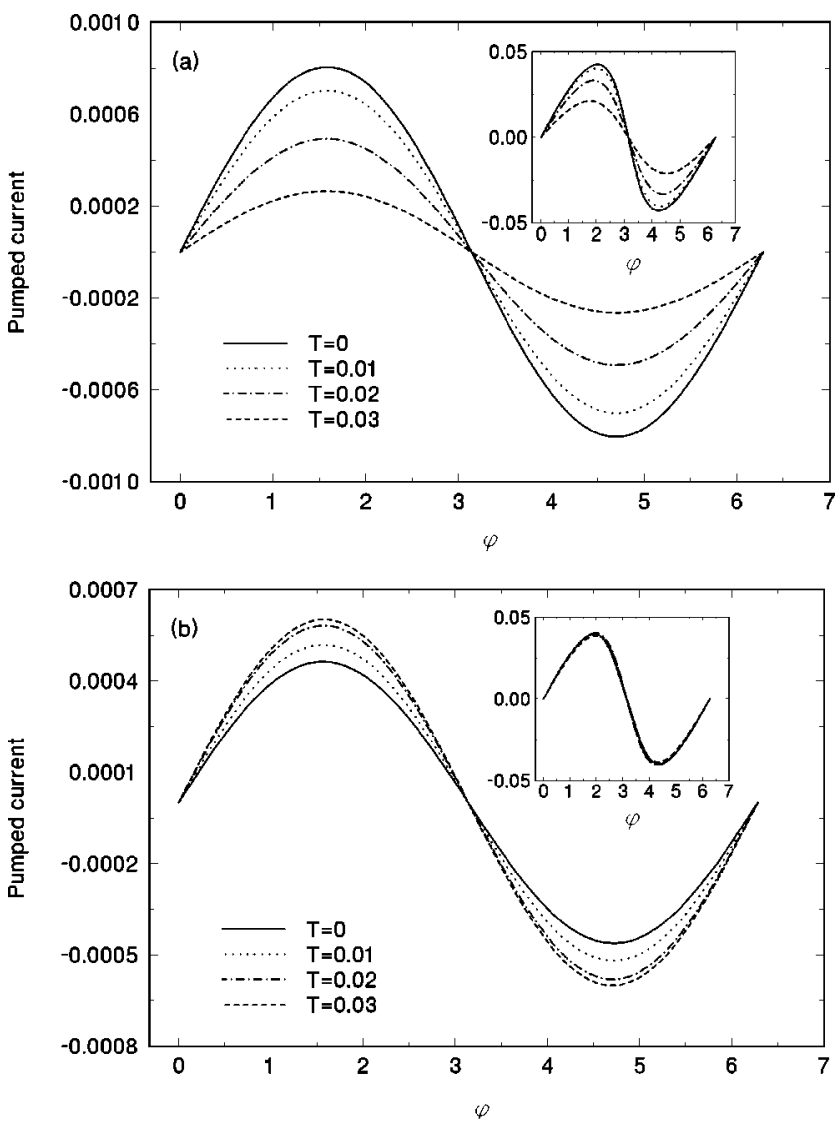

FIG. 4. The pumped current versus phase difference at different temperatures. (a). Main figure: $v_{g}=-2.5$ and $v_{p}=0.01 v_{0}$; inset: $v_{g}=-2.5$ and $v_{p}=0.1 v_{0}$. (b). Main figure: $v_{g}=-0.48$ and $v_{p}$ $=0.01 v_{0}$; inset: $v_{g}=-0.48$ and $v_{p}=0.1 v_{0}$. 
current is bilinear in pumping amplitude in the weak pumping regime. ${ }^{2}$ For larger amplitude $v_{p} / v_{0}>0.03$ it is almost linear with different slopes depending on temperatures. The slope is smaller at higher temperature. For the gate voltage near the resonant level, the pumped current has similar behavior except that it is not sensitive to the change of temperature. Figure 4 displays the pumped current as a function of phase difference $\phi$ between two pumping forces for different pumping amplitudes. The pumped current is antisymmetric about the phase difference $\phi=\pi$. In the weak pumping regime $\left(v_{p}=0.01 v_{0}\right)$, the pumped current shows the sinusoidal behavior and peaked at $\phi=\pi / 2$. This is because in the weak pumping regime, the pumped current is bilinear in the pumping amplitude and proportional to $\sin \phi .{ }^{2}$ In the strong pumping regime $\left(v_{p}=0.1 v_{0}\right)$, we start to see nonsinusoidal behavior as higher-order terms of pumping amplitude come into play. The maximum pumped current occurs approximately at $\phi=0.6 \pi$ [see Figs. 4(a) and 4(b)]. Similar nonlinear behavior is also seen experimentally ${ }^{3}$ although the physical origin may be different.

In summary, we have studied the parametric electron pumping through a quantum dot in the Kondo regime using a nonequilibrium Green's-function theory. We found that the behavior of the pumped current is closely related to the conductance. As one varies the pumping amplitude, the pumped current increases quadratically for small amplitude and then scales linearly with the pumping amplitude. Because of the resonant nature of the pumping, the pumped current shows nonsinusoidal dependence on the phase difference of the pumping parameters. In this paper, we have used the adiabatic theory to calculate the pumped current. This theory is valid in the low-frequency regime and cannot account for the anomaly at $\phi=\pi$ found experimentally. ${ }^{3}$ At finite frequency, one must use the real-space nonequilibrium Green's-function method $^{14}$ to calculate the pumped current.

We gratefully acknowledge the support by RGC of Hong Kong SAR under grant number HKU 7091/01P.
${ }^{1}$ D.J. Thouless, Phys. Rev. B 27, 6083 (1983).

${ }^{2}$ P.W. Brouwer, Phys. Rev. B 58, R10 135 (1998).

${ }^{3}$ M. Switkes, C. Marcus, K. Capman, and A.C. Gossard, Science 283, 1905 (1999).

${ }^{4}$ F. Zhou, B. Spivak, and B.L. Altshuler, Phys. Rev. Lett. 82, 608 (1999).

${ }^{5}$ I.L. Aleiner, B.L. Altshuler, and A. Kamenev, Phys. Rev. B 62, 10373 (2000).

${ }^{6}$ Y.D. Wei, J. Wang, and H. Guo, Phys. Rev. B 62, 9947 (2000).

${ }^{7}$ M.G. Vavilov, V. Ambegaokar, and I.L. Aleiner, Phys. Rev. B 63, 195313 (2001).

${ }^{8}$ P.W. Brouwer, Phys. Rev. B 63, 121303 (2001).

${ }^{9}$ P. Sharma and C. Chamon, Phys. Rev. Lett. 87, 096401 (2001)

${ }^{10}$ I.L. Aleiner and A.V. Andreev, Phys. Rev. Lett. 81, 1286 (1998).

${ }^{11}$ Y.D. Wei, J. Wang, H. Guo, and C. Roland, Phys. Rev. B 64, 115321 (2001)

${ }^{12}$ T.A. Shutenko, I.L. Aleiner, and B.L. Altshuler, Phys. Rev. B 61, 10366 (2000).

${ }^{13}$ J. Wang, Y.D. Wei, B.G. Wang, and H. Guo, Appl. Phys. Lett. 79, 3977 (2001); J. Wang and B.G. Wang, Phys. Rev. B 65, 153311 (2002).
${ }^{14}$ B.G. Wang, J. Wang, and H. Guo, Phys. Rev. B 65, 073306 (2002).

${ }^{15}$ M. Moskalets and M. Buttiker, Phys. Rev. B 64, 201305 (2001); cond-mat/0201259.

${ }^{16}$ A.P. Jauho, N.S. Wingreen, and Y. Meir, Phys. Rev. B 50, 5528 (1994).

${ }^{17}$ M. Buttiker, H. Thomas, and A. Pretre, Z. Phys. B: Condens. Matter 94, 133 (1994).

${ }^{18}$ B.G. Wang, J. Wang, and H. Guo, J. Appl. Phys. 86, 5094 (1999).

${ }^{19}$ A. Levy Yeyati, A. Martin-Rodero, and F. Flores, Phys. Rev. Lett. 71, 2991 (1993).

${ }^{20}$ H. Kajueter and G. Kotliar, Phys. Rev. Lett. 77, 131 (1996).

${ }^{21}$ Y. Meir, N.S. Wingreen, and P.A. Lee, Phys. Rev. Lett. 70, 2601 (1993); T.K. Ng, ibid. 76, 487 (1996); Q.F. Sun, H. Guo, and T.H. Lin, ibid. 87, 176601 (2001).

${ }^{22}$ T. Aono, M. Eto, and K. Kawamura, J. Phys. Soc. Jpn. 67, 1860 (1998); P. Schwab and R. Raimondi, Phys. Rev. B 59, 1637 (1999); R. Aguado and D.C. Langreth, Phys. Rev. Lett. 85, 1946 (2000); R. Lopez, R. Aguado, G. Platero, and C. Tejedor, ibid. 81, 4688 (1998).

${ }^{23}$ N.S. Wingreen and Y. Meir, Phys. Rev. B 49, 11040 (1994).

${ }^{24}$ Similar to Ref. 20, we have assumed that there is no magnetic symmetry breaking $\left(n_{\sigma}=n_{\sigma}=n\right)$. 